\title{
Geruchsbezeichnungen in der Übersetzung am Beispiel des Romans Das Parfum. Die Geschichte eines Mörders von Patrick Süskind
}

\author{
The Translation of smell lexemes used \\ in the novel Perfume: The Story of a Murderer \\ by Patrick Süskind
}

ABSTRACT. The aim of the following article is to present the results of the translation of smell lexemes used in the Novel Parfume: The Story of a Murderer by Patrick Süskind and their Polish equivalents in Pachnidło. Historia pewnego mordercy by Małgorzata Łukasiewicz. The article discusses semantic, lexical and syntactical problems, influence of the context on the translation and the results of the analysis.

KEYWORDS: translation criticism, semantics, morphology, syntax, context, translation analysis.

\section{DARSTELLUNG DER PROBLEMATIK}

Die Verbalisierung von Gerüchen ist immer mit dem Problem verbunden, wie die Wahrnehmungen in die Sprache umgesetzt werden sollten, weil sie als momentane olfaktorische Wahrnehmungen nicht zu behalten sind. Obwohl sie mit verschiedenen Worten, mithilfe von Beschreibungen oder Metaphern wiedergegeben werden, ist jedoch nicht zu leugnen, dass sie sich linguistisch gesehen äußerst schwer versprachlichen lassen. Da die in einzelnen Sprachen für die Beschreibung der olfaktorischen Wahrnehmungen zur Verfügung stehenden Ausdrucksmittel mangelhaft sind, führen 
sie bei der Übersetzung von Geruchsbezeichnungen vor allem in literarischen Werken zu Abweichungen von dem Originaltext. Aus diesem Grunde stellt sich dieses Problem interesseerweckend dar.

Für die Übersetzungsanalyse von Nominativen aus dem deutschen Wortfeld der Geruchsbezeichnungen und ihren polnischen Äquivalenten wurden die Belege ${ }^{1}$ aus dem Roman Das Parfum. Die Geschichte eines Mörders von Patrick Süskind und der polnischen Übersetzung von Małgorzata Łukasiewicz unter dem Titel Pachnidło. Historia pewnego mordercy entnommen.

In erster Linie sollten die Fragestellungen zur literarischen Kritik, Rolle des Übersetzers, die Probleme der semantischen, morphologischen und syntaktischen Natur in der Übersetzung diskutiert werden, um zu erschließen, ob die lexikalischen Ausdrucksmittel der gegebenen Geruchsbezeichnung im jeweiligen Sprachsystem zu finden sind. Dabei sollten aus kontrastiver Sicht die Komponentenanalyse der deutschen und polnischen Geruchsbezeichnungen Anwendung finden und ihre Äquivalenzbeziehungen berücksichtigt werden.

\section{KRITIK DES LITERARISCHEN ÜBERSETZENS}

Bei jeder literarischen Übersetzung wird der Übersetzer in einem Normenkonflikt widerstreitender ausgangs- und zielseitiger Faktoren und Konventionen stehen. Es wurde bereits darauf hingewiesen, dass die Übersetzung mit einem Entscheidungsprozess verbunden ist. Trotzdem stellt jede literarische Übersetzung eine motivierte Reaktion des Übersetzers auf literarische und kulturelle Gegebenheiten dar.

\subsection{Semantik: Die Bedeutung semantischer Bündelungen im Übersetzungsprozess}

„Die Sprache wechseln, heißt in eine andere Welt wechseln" (Salnikov 1995: 1). In übersetzungstheoretischen Beiträgen sind jedoch Aussagen zu finden, dass beim Übersetzungsakt viele Aspekte berücksichtigt werden sollten. Daher hat eine Übersetzung eine Verknüpfung kulturspezifischer Konstellationen, Sprach- und Textformen, Inhalt und Sinn darzustellen. Von

\footnotetext{
${ }^{1}$ Die im Beitrag präsentierten Ergebnisse stützen sich auf die aus der Originalfassung des Romans und seiner polnischer Übersetzung exzerpierten 185 Belege.
} 
daher ist Übersetzen nach Siever (2010: 43) „,komplexes Gefüge von Entscheidungsprozessen" genannt, in dem dem Übersetzer die Forderung gestellt wird, nach dem richtigen Lexem zu suchen. Dabei erweisen sich die Erkenntnisse der strukturellen Semantik relevant, weil sie besagen, dass sich die „lexikalischen Systeme zweier Sprachen in vielen Fällen nicht entsprechen und dass es demzufolge Bedeutungsüberlappungen gibt" (Snell-Hornby, Hönig, Kußmaul, Schmitt 2006: 49). Die Wortbedeutungen sind nicht als statisch zu betrachten, sondern ihre Bedeutungen von einem Kontext determiniert oder sogar geschaffen werden können. Nida vertritt daher die These, dass die Aufgabe des Übersetzers nicht darin besteht, die Wörter, sondern Bündelungen semantischer Merkmale zu übersetzen, mit derer Übersetzung die ausgangssprachlichen Wortformen sowie ihre Bedeutungen gelöst und in andere zielsprachliche abstrahierte Bedeutungen paraphrasiert werden (Snell-Hornby et al. 2006: 49).

\subsection{Morphologie als Übersetzungschwierigkeit}

In jeglichen Übersetzungen sollten außer dem Inhalt die Oberflächenstrukturen des Originaltextes beibehalten werden, wobei „die 'Wörtlichkeit' als die bei denotativer Äquivalenz und zielsprachlicher grammatischer Korrektheit größtmögliche formale Ähnlichkeit von Ausgangs- und Zieltext" (Kittel, Frank, Greiner, Hermans, Koller, Lambert, Paul 2004: 466) festgestellt werden kann. Übersetzungstheoretiker Wilss (1977: 202-203, zit. n. Kittel et al. 2004: 466) präzisiert, dass man mit einer Übersetzungsschwierigkeit zu tun haben kann, "wo ausgangssprachliches Textverständnis vorausgesetzt, eine lexikalische, syntagmatische oder syntaktische Eins-zu-Eins-Entsprechung zwischen ausgangs- und zielsprachlichem Textsegment nicht möglich ist".

Es ist offensichtlich, dass das Erkennen morphosyntaktischer Übersetzungsschwierigkeiten im Bereich der Übersetzerkompetenz liegt. Dies ermöglicht dem Übersetzer, sprachliche Fehlleistungen im Übersetzungsakt zu vermeiden und damit die Qualität des übersetzten Textes zu verbessern.

\subsection{Syntax: Die Korrespondenz- und Präferenzregeln als Schwierigkeit im Translationsvorgang}

Erst bei Betrachtung der Syntax als eines übersetzungsrelevanten Problems wird klar, dass in der Syntax "die syntagmatischen Beziehungen in Sätzen“ (Snell-Hornby et al. 2006: 53) beschrieben und erklärt werden und 
ihre Grundeinheiten „syntaktische Wörter, die nach verschiedenen Strukturprinzipien organisiert werden können" (Snell-Hornby et al. 2006: 53) sind. Bei einer Texttransformation von einer Ausgangssprachhandlung zur Zielsprachhandlung, die von Ausgangs- und Zieltext realisiert werden, sind zwei Arten von syntaktischen Regeln zu berücksichtigen und zwar die nach Snell-Hornby et al. (2006: 53). sogenannten Korrespondenz- und Präferenzregeln. Übersetzungsrelevante Probleme können bei den syntaktischen Regeln für bestimmte Textbereiche und/oder -formen präferentiellen Verwendungsregeln zum Vorschein kommen. Darunter werden hier die Regeln verstanden, die "bei der intentional-sozialen Wahl von syntaktischen Alternativen" (Snell-Hornby et al. 2006: 54) charakteristisch und bei der Übersetzung nicht nur einzelsprachlich sondern auch aus kontrastiver Sicht beschrieben werden sollten.

$\mathrm{Zu}$ den translatorisch relevanten Präferenzregeln, die im Translationsvorgang Schwierigkeiten bereiten können, gehört Snell-Hornby et al. (2006: 54) zufolge der im Deutschen besonders in fachsprachlichen Sprachhandlungen dominierende Nominalstil, dessen deutliche Verwendungstendenz auch im Roman Süskinds festgestellt werden kann.

\subsection{Kontext im Übersetzungsprozess}

Bei literarischer Kritik spielt natürlich der Kontext eine nicht weniger bedeutsame Rolle sowie Disambiguierung und schließlich Wahl des Übersetzers zwischen Kontext und Monosemierung. Obwohl Leisi behauptet, dass das Wort das wichtigste Element eines Sprachsystems ist, weist Christiane Nord darauf hin, dass „ein Text eine Einheit ist, auf die es beim Übersetzen ankommt, weil er als Ganzes vom Empfänger wahrgenommen und rezipiert wird" (Nord 1993: 23). Die Wörter in einem Text verleihen mit ihrer jeweiligen Kode-Bedeutung einander Kontext. Die Wörter können jedoch aufgrund vieler Bedeutungsrelationen viele Bedeutungen haben. Um die Polysemie einer Bedeutungseinheit ausschließen zu können, kann das Phänomen der Disambiguierung behilflich sein, d.h. „Vorgang und Ergebnis der Auflösung lexikalischer oder struktureller Mehrdeutigkeit sprachlicher Ausdrücke durch den sprachlichen oder außersprachlichen Kontext" (Bußmann 2002: 169). Dadurch, dass der Kontext die Bedeutung den Wörtern verleiht, steht ein Übersetzer immer vor der Wahl, ein entsprechendes Lexem zu finden, um das nach Stolze „Unbestimmte, Polyvalente im Originaltext für die Deutungserfahrung des Lesers der Übersetzung zu bewahren" (Stolze 1985: 355). 


\section{3. ÜBERSETZUNGSANALYSE AUSGEWÄHLTER GERUCHSBEZEICHNUNGEN DES ROMANS DAS PARFUM. DIE GESCHICHTE EINES MÖRDERS}

Aus dem Belegmaterial wurde ersichtlich, dass aus dem deutschen nominalen Wortfeld Geruchsbezeichnungen 8 Lexeme zum Vorschein kamen und zwar: Geruch, Duft, Gestank, Dunst, Odeur, Brodem, Ausdünstung und Dampf, deren Übersetzungen strikt kontextabhängig waren.

Bei dem Vergleich deutscher und polnischer Geruchsbezeichnungen wurde das Problem der Versprachlichung von Geruchswahrnehmungen besprochen, weil es unumstritten ist, dass ihre Umwandlung in die sprachlichen Äußerungen Schwierigkeiten bereitet. Der Grund hierfür ist einerseits darin zu sehen, dass wir gezwungen sind, auf Vokabular aus verschiedenen Sprachbereichen zurückzugreifen und auf der anderen Seite wie Staniewski behauptet „nicht nur in der Semantik dieser Wortschatzeinheiten zu suchen [sind], sondern auch in der Eigenart des zu betrachtenden Lebensbereichs, was ihre unterschiedliche Wiederspiegelung (sic!) in den beiden Sprachen hat" (2010: 265).

In der Übersetzunganalyse wurden 8 Geruchsbezeichnungen und ihre polnischen Entsprechungen untersucht. Das Diversifikationsverhältnis (Eins-zu-viele Entsprechung), in dem einem AT-Lexem mehrere ZT-Lexeme zugeordnet werden können, kann beim Übersetzen Schwierigkeiten bereiten. Deswegen entscheidet der Übersetzer, welches Lexem in welcher kontextabhängigen Situation zu verwenden ist. Welche Lösungen für die genannten Probleme Łukasiewicz gefunden hat, wird in folgenden Beispielen beleuchtet. Der folgende Beleg betrifft das Lexem Geruch:

(1) ... heute in Vergessenheit geraten ist so sicher nicht deshalb, weil Grenouille diesen nicht berühmten Finstermännern an Selbstüberhebung, Menschenverachtung, Immoralität, kurz an Gottlosigkeit nachgestanden hätte, sondern weil sich sein Genie und sein einziger Ehrgeiz auf ein Gebiet beschränkte, welches in der Geschichte keine Spuren hinterläßt: auf das flüchtige Reich der Gerüche (Par, 5).

... popadło dziś w zapomnienie, to z pewnością nie dlatego, iżby Grenouille ustępował owym głośniejszym niegodziwcom w pysze, pogardzie dla ludzi, braku moralnych zasad, jednym słowem - w bezbożności, lecz dlatego, że geniusz jego i jedyna ambicja ograniczały się do dziedziny, która w historii nie pozostawia śladów: do ulotnego królestwa zapachów (Pach, 5).

Aus der Komponentenanalyse geht hervor, dass das Lexem Geruch entweder angenehm oder übel, intensiv oder subtil sein kann. Bezüglich semantischer Merkmale entspricht das polnische Lexem zapach dem deutschen Geruch. Im Polnischen kann Geruch sowohl mit zapach als auch mit woń über- 
setzt werden (WSNP). Der Beleg (1) ist ein Abschnitt des Textes, dessen Übersetzung dem Original entspricht. Der Autor benutzt die Pluralform Gerüche. Dieselbe Form ist in der Übersetzung wiederzufinden. Die deutsche und polnische Geruchsbezeichnung wird neutral assoziiert.

Ein weiters Beispiel schildert das Lexem Duft. DUW zerlegt das deutsche Lexem Duft folgendermaßen: 'angenehm empfundener, zarter bis intensiver Geruch'. Die Komponentenanalyse zeigt, dass Duft entweder subtil oder intensiv, aber immer angenehm ist. Dem Duft stehen polnische Entsprechungen zapach, aromat, woń entgegen (WSNP). Zapach und woń weisen andere Merkmale als Duft auf. Aromat dagegen ist dem Duft nicht nur in der Definition ähnlich: 'przyjemny zapach, miła woń' (SJP), ['angenehmer Geruch; angenehmer Duft'] sondern löst auch ähnliche Konnotationen aus.

(67) Ihm schwante sonderbar, dieser Duft sei der Schlüssel zur Ordnung aller anderen Düfte, man habe nichts von den Düften verstanden, wenn man diesen einen nicht verstand, und er, Grenouille, hätte sein Leben verpfuscht, wenn es ihm nicht gelänge, diesen einen zu besitzen (Par, 50).

Miał dziwne uczucie, że zapach ten jest kluczem do porządku wszystkich innych zapachów, że nie można mieć pojęcia o zapachach, jeśli nie pojmie się tego jednego, a on, Grenouille, będzie miał spartaczone życie jeśli nie uda mu się posiąść tej woni (Pach, 41).

Im dargebotenen Beleg wird über Grenouilles ersten gestohlenen Geruch gesprochen. Um seine Unwiderstehlichkeit auszudrücken, benutzte Süskind vor allem das angenehm konnotierte Lexem Duft. Im Zieltext werden jedoch zapach und woń eingeführt, womit nicht zum Ausdruck gebracht wurde, dass der Geruch so einmalig war, dass es Grenouille zum Mord bewogen hat, weil die Lexeme für neutrale Assoziationen sorgen. Sie hätte vielleicht aromat un woń verwenden können.

Ein anderer Beleg stellt den Gebrauch der polnischen Bedeutungseinheit substancja dar.

(114) Er stürzte eine Glasplatte auf die Wanne, zog den Rest des Duftes auf zwei Fläschchen, die er mit Etiketts versah, darauf schrieb er den Namen $>$ Nuit Napolitaine $<$ (Par, 112).

Położył na wanience szklaną płytkę, resztę substancji przelał do dwóch flaszeczek, umieścił na nich etykietki i wpisał nazwę: „Noc Neapolitańska“ (Pach, 89).

Im Beleg (114) wird der vom Grenouille wahrgenommene Duft durch das polnische Lexem substancja ersetzt. Die Übersetzerin spricht von einer realen Flüssigkeit, was durch die definitorische Erklärung untermauert 
wird, weil substancja 'jednorodną materią o ściśle określonym składzie chemicznym' (SJP), ['eine einheitliche Substanz mit einer genauen bestimmten chemischen Zusammensetzung'] ist. An dieser Stelle konnte die Übersetzerin den polnischen Äquivalenten des Duftes nicht treu bleiben, weil der Kontext es nicht zum Ausdruck bringen kann.

Interessant ist die Erscheinung im Beleg, bei dem die polnische Geruchsbeschreibung des Verbs pachnieć benutzt wird, was dem deutschen Verb duften entspricht.

(71) Die Straße duftete nach den üblichen Düften von Wasser, Kot, Ratten und Gemüseabfall (Par, 53).

Ulica pachniała zwyczajnie wodą, łajnem, szczurami i odpadami jarzyn (Pach, $42-43)$.

Nach DUW befindet sich die Bedeutung der Einheit duften auf einer neutralen Ebene - 'Duft verbreiten; einen bestimmten oder für etwas charakteristischen Duft verbreiten' (DUW). Erst in Verwendungsbeispielen kann geschlussfolgert werden, dass duften positiv assoziiert ist und mit Lexemen vorkommt, die den Geruch angenehm bewerten, z.B. 'die Rosen duften stark' (DUW). Um den Geruch in den Straßen zu versprachlichen, benutzte Süskind den Ausdruck die Straße duftete nach den üblichen Düften. Erst die folgenden Nomina Wasser, Kot, Ratten und Gemüseabfall verleihen den unangenehmen sinnlichen Eindruck. Łukasiewicz schrieb dagegen ulica pachniała, was im Polnischen auf Anhieb wohltuend konnotiert ist. Ihre Lösung wirkt verwirrend in Bezug auf den Kontext. Die Verbindung von pachnieć mit den Nomina woda, tajnem, szczurami i odpadami jarzyn kommt an dieser Stelle in Frage, weil es sinnvoller ist, das Verb tracić oder śmierdzieć zu benutzen, um die widerliche Sinnwahrnehmung zu vermitteln.

Das weitere Beispiel für ungetreue Wiedergabe ist bei dem Lexem Dunst zu sehen. Im Polnischen stehen für die Realisierung besonders viele Lexeme gegenüber: para, opary, opar, mgła, wyziew, czad (WSNP). Die Wahl der Übersetzerin im folgenden Beleg ist anzweifelbar.

(177) Er roch sie jetzt einzeln, er roch sogar den Dunst ihrer Pferde (Par, 270).

Teraz zwęszył ich wyraźnie, dobiegł go nawet zapach potu ich wierzchowców (Pach, 212).

Dunst ihrer Pferde hat sie mit zapach potu ich wierzchowców übersetzt. Im Ausgangstext wird vom Schweiß der Pferde nicht gesprochen. Es wird angenommen, dass die Übersetzerin den Ausdruck zapach potu ich wierzchowców mit Absicht verwendet, um den Gebrauch negativer Geruchsbezeichnungen zu meiden. Als Gegenargument für ihre Wahl ist die definitorische Erklä- 
rung des Lexems wyziew anzuführen: 'zwykle w $1 \mathrm{~m}$., woń (zwykle nieprzyjemna) ulatniająca się z ogrzanych cieczy lub ciał stałych' (SJP), ['meistens im Pl., ein (meistens unangenehmer) sich aus erhitzten Flüssigkeiten oder Festkörper ausströmender Duft']. Hätte die Übersetzerin wyziewy ich wierzchowców geschrieben oder sich des negativ konnotierten Lexems odór bedient, wäre die Übersetzung dem Original in seinem Grundton beständiger.

Die Übersetzungsanalyse von den Lexemen Gestank, Ausdünstung, Brodem, Dampf und Odeur hat ähnliche Unstimmigkeiten aufgezeigt. Die Abweichungen und Zusammenstöße der Übersetzerin gegen die angenommenen Ausgangsregeln, die Wörterbuchdefinitionen und die polnischen Entsprechungen der Ausgangstextlexemen haben zu vielen Schlussfolgerungen geführt.

\section{SCHLUSSFOLGERUNGEN}

Der unternommene Versuch, die deutschen Geruchsbezeichnungen und ihre polnischen Entsprechungen zu untersuchen, lässt folgendes Resümme zu: Aus der Analyse ist hervorgegangen, dass die angenommene These, die Übersetzung sei eine Interpretation, bestätigt wurde. Die Übersetzerin des Romans erwies sich als eine Person, die den Text interpretiert und ihr Bild des Verstandenen dargestellt hat. Die getroffene Wahl hat zur Folge, dass in den Belegen Unstimmigkeiten zwischen Original und Übersetzung und sogar Übersetzungsfehler ermittelt wurden. Ihr mangelhaftes Ausdrucksvermögen kann der Grund dafür sein, dass sie an einigen Stellen nicht im Stande war, die von Süskind verwendeten Geruchsbezeichnungen wortwörtlich im Polnischen auszudrücken, sondern hier zu anderen wie substancja griff oder für ausgangssprachliche Geruchseindrücke Konkretisierungen vorgenommen hat wie z.B. für das Lexem Duft als Bezeichnung der Flüssigkeit. Unumstritten erwies sich die These, dass der Bedeutungsbegriff eines Wortes vom Kontext abhängig ist. Viele Begriffe haben dadurch, dass sie in einem bestimmten Kontext und in der Situation Bedeutungsrelationen mit anderen Ausdrücken eingehen, eine andere - dem Wortfeld spezifische Bedeutung gewonnen (bspw. substancja). Die Abweichungen der polnischen Äquivalente für die Geruchsbezeichnungen sind einerseits von der Wortrekurrenz und andererseits von den Konfliktlösungen zwischen Monosemierung und Wahl des Übersetzers abhängig und auf die bewussten Entscheidungen der Übersetzerin zurückzuführen.

Die durchgeführte Analyse hat gezeigt, dass die Übersetzung hinsichtlich der Morphologie dem Original nicht so treu geblieben ist. Die Annahme, dass die größtmögliche formale und grammatische Korrektheit bei literari- 
schen Texten festgestellt werden sollte, spiegelt sich bei Łukasiewicz nicht wider. Obwohl sie die morphosyntaktischen Übersetzungsschwierigkeiten in der Regel erkannte und den Übersetzungsfehler vermied, kommen in Pachnidło. Historia pewnego mordercy viele Übersetzungsunstimmigkeiten zum Vorschein. Dieses betrifft vor allem den Gebrauch der Plural- und Singularform von nominalen Geruchsbezeichnungen. Da in der Übersetzung häufig statt der ausgangssprachlichen Singularform im Zieltext die Pluralform des gegebenen Lexems benutzt wurde, wird vermutet, dass damit die Funktion zur Intensivierung der Geruchseindrücke erfüllt werden sollte.

Das leitende Motiv der Übersetzungsanalyse war die Annahme, dass die deutschen Geruchsbezeichnungen in einem Übersetzungsprozess Schwierigkeiten bereiten. Eine dominierende Rolle im Übersetzungsprozess sowie im Konflikt zwischen Wortrekurrenz einerseits und Kollokationen oder Disambiguierung andererseits nimmt die Übersetzerin ein. Pachnidto. Historia pewnego mordercy ist ihre Interpretationsweise. Die von ihr gebrauchten Geruchsbezeichnungen geben dem Leser ein viel negativeres Bild vom damaligen Paris und von den dort herrschenden Gerüchen als das Original.

Voraussetzung für die Untersuchung war, dass die Übersetzerin für die Geruchsbezeichnungen aus dem deutschen nominalen Wortfeld die polnischen Nomina verwendet. Es wurde jedoch festgestellt, dass sowohl die Rekurrenz als auch die Geruchsdarstellungen von den verbalen Geruchsbezeichnungen in der Übersetzung beeinflusst wurden (bspw. pachnieć).

Es wurde darauf hingewiesen, dass Süskind zur Geruchsdarstellung meistens positiv oder neutral konnotierte Lexeme benutzt. Das Paradox ist, dass er sie vor allem in negativ spezifizierten Kontexten verwendet (Duft des schweißenden Pferdes). Diese Dichotomie üble vs. schöne Gerüche bietet der Übersetzerin die Möglichkeit mit den Gerüchen zu jonglieren. Der Zusammenstoß von Gestank vs. Parfum wird in der Übersetzung nicht ersichtlich, weil die Übersetzerin den Kontext eindeutig negativ darstellt. Im Polnischen gibt es mehr Lexeme der Geruchsbezeichnungen als im Deutschen, wodurch zu erwarten war, dass der Spielraum für die Übersetzung deutlich größer ist. In der Übersetzung ist dieser Spielraum kleiner ausgefallen, weil die Übersetzerin weder die Rekurrenz noch die Verwendung eines positiven Lexems in den negativen Kontexten erhalten hat, wodurch die Vermittlung der spezifischen Dichotomie Gestank vs. Parfum gestört wurde.

Zusammenfassend lässt sich feststellen, dass die Übersetzung nicht so treu angefertigt ist, wie es möglich gewesen wäre. Aus den aufgeführten Unterschieden resultiert, dass in der Übersetzung an einigen Stellen andere Geruchseindrücke als die im Werk Süskinds zum Ausdruck gebracht wurden. Die zielsprachliche Fassung ist ein Kompromiss der Übersetzerin, die die Überlegung anstellen musste, welcher Aspekt des Ausgangstextes am 
vollständigsten zu erhalten ist und bei welchen Aspekten Einbußen in Kauf genommen werden können. Außer mangelnder Kompetenz des Ausdrucksvermögens eines Übersetzers, Verstehensschwierigkeiten, sprachlich syntaktischer, morphologischer und semantischer Übersetzungsschwierigkeiten ist schließlich die Tiefenvermutung des Übersetzers, wo im Originaltext ein konventioneller Ausdruck steht, die häufigste Ursache für Übersetzungsfehler, was im Falle von Pachnidło. Historia pewnego mordercy zur Folge hat, dass den quantitativen Analyseergebnissen zufolge fast ein Fünftel (38 von 254 analysierten Geruchsbezeichnungen) anders übersetzt ist, als es hätte übersetzt werden müssen.

\section{LITERATURVERZEICHNIS}

Bußmann, H., 2002. Lexikon der Sprachwissenschaft. Stuttgart: Alfred Kröner Verlag.

Doroszewski, W., 1996. Stownik jezzyka polskiego. Warszawa: Wydawnictwo Naukowe PWN $(=\mathrm{SJP})$.

Kittel, H., Frank, A.,P., Greiner, N., Hermans, T., Koller, W., Lambert, J., Paul, F., 2004. Übersetzung, Translation, Traduction. Berlin: Walter de Gruyter.

Kunkel-Razum, K., Scholze-Stubenrecht, W., Wermke, M., 2007. Deutsches Universalwörterbuch 6. Auflage. Mannheim-Leipzig-Wien-Erich: Dudenverlag (=DUW).

Nord, Ch., 1993. Einführung in das funktionale Übersetzen. Tübingen-Basel: A. Francke

Piprek, J., Ippoldt, J., 2001. Wielki stownik niemiecko-polski. Warszawa: Wiedza Powszechna PW (=WSNP).

Salnikov, N., 1995. Sprachtransfer - Kulturtransfer. Frankfurt am Main: Peter Lang GmbH.

Siever, H., 2010. Übersetzen und Interpretation. Frankfurt am Main: Peter Lang GmbH.

Snell-Hornby, M., Hönig, H.,G., Kußmaul, P., Schmitt, P.A., 2006. Handbuch Translation. Tübingen: Stauffenburg Verlag Brigitte Narr GmbH.

Staniewski, P., 2010. Schwierigkeiten bei der Übersetzung der Geruchsbezeichnungen am Beispiel des Romans Das Parfum von Patrick Süskind. In: Małgorzewicz, A. (Hrsg.). Translation: Teorie-Praxis-Didaktik. Dresden-Wrocław: Neisse Verlag, ATUT, 259-267.

Stolze, R., 1985. Grundlagen der Textübersetzung. Heidelberg: Julius Gross Verlag.

Süskind, P., 1990. Pachnidto. Historia pewnego mordercy. Aus dem Deutschen von Małgorzata Łukasiewicz. Warszawa: Świat książki (=Pach).

Süskind, P., 1994. Das Parfum. Die Geschichte eines Mörders. Zürich: Digenes Verlag AG (=Par). 